\title{
Article
}

\section{Methods for speeding up the Boundary Element Solution of Acoustic Radiation Problems}

Kirkup, Stephen Martin and Henwood, D J

Available at http://clok.uclan.ac.uk/7316/

Kirkup, Stephen Martin ORCID: 0000-0002-9680-7778 and Henwood, D J (1992) Methods for speeding up the Boundary Element Solution of Acoustic Radiation Problems. ASME Journal of Vibration and Acoustics, 114 (3). pp. 374380. ISSN 0739-3717

It is advisable to refer to the publisher's version if you intend to cite from the work. http://dx.doi.org/10.1115/1.2930272

For more information about UCLan's research in this area go to http://www.uclan.ac.uk/researchgroups/ and search for <name of research Group>.

For information about Research generally at UCLan please go to http://www.uclan.ac.uk/research/

All outputs in CLoK are protected by Intellectual Property Rights law, including Copyright law. Copyright, IPR and Moral Rights for the works on this site are retained by the individual authors and/or other copyright owners. Terms and conditions for use of this material are defined in the policies page.

\section{CLoK}

Central Lancashire online Knowledge www.clok.uclan.ac.uk

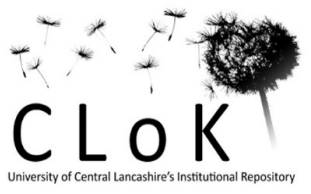




\section{Methods for Speeding Up the \\ S. M. Kirkup \\ Department of Mathematics \\ and Computer Science, \\ University of Salford. \\ Salford, United Kingdom \\ D. J. Henwood \\ Department of Mathematical Sciences, \\ Brighton Polytechnic, \\ Brighton, United Kingdom Boundary Element Solution of Acoustic Radiation Problems \\ Methods for speeding up the boundary element solution of acoustic radiation prob- lems are considered. The methods are based on solving the integral equation for- mulation of Burton and Miller for the exterior Helmholtz equation over a range of frequencies simultaneously. Methods for speeding up the computation of the discrete forms of the integral operators and the solution of the linear systems that arise in forms of the integral operators and the solution of the linear systems that arise in
the boundary element method are considered. A particular implementation of speed- up methods is described. Results from the application of this to test problems are given.}

\section{Introduction}

A set of vibrating objects in an acoustic medium such as air or water produces an acoustic field. The problem of determining the properties of the three-dimensional acoustic field from a description of the vibration of the objects, whatever their shape and the nature of their vibration, is the acoustic radiation problem (ARP) we consider in this paper. The overall problem is that of developing a computer program which accepts a description of the surfaces and their vibration and, from that, is able to compute satisfactory approximations to the acoustic properties.

The ARP is modelled by a set of closed vibrating bodies surrounded by an infinite acoustic medium, as illustrated in Fig. 1; this is known as the acoustic radiation model (ARM). The symbol $D$ is used to represent the points in the interior regions, $S$ to represent the points on the boundaries, and $E$ to represent the points in the exterior region.

Acoustic problems are governed by the wave equation. The decomposition of the time variables into Fourier components reduces the ARM to a sequence of three-dimensional exterior Helmholtz problems with Neumann boundary conditions (HE3N problems) of the form

$$
\begin{gathered}
\nabla^{2} \varphi(p)+k^{2} \varphi(p)=0 \quad(p \in E) \\
\frac{\partial \varphi}{\partial n_{p}}(p)=v(p) \quad(p \in S) ;(v(p) \text { given }) \\
\operatorname{limit}_{r \rightarrow \infty} r\left\{\frac{\partial \varphi}{\partial r}-i k \varphi\right\}=0
\end{gathered}
$$

for $k$ (the wavenumber) taking the values $k_{1}, k_{2}, \ldots, k_{n}$. In the majority of practical situations the time-dependent variables can only be described approximately by a finite linear sum of Fourier components and in order to achieve a satisfactory

Contributed by the Technical Committee on Vibration and Sound for publication in the Journal of VIrRation and Acoustics. Manuscript received Jan. 1991. Associate Technical Editor: R. F. Keltie.

374 I Vol. 114, JULY 1992 approximation a large number of distinct wavenumbers are often used [see, for example, Skudrzyk (1971) pp. 390-391].

The ARM can be formulated in terms of a boundary integral equation (BIE). Numerical methods based on the BIE are known as boundary element methods (BEMs). The use of the $\mathrm{BEM}$ as a means of obtaining a numerical solution of acoustic radiation and scattering problems has been investigated by researchers over the past two or three decades. Difficulties in the achievement of a robust method and in its implementation have hindered its use. However, with the introduction of the method of Schenck (1968), with the improved integral equation formulations introduced by Kussmaul (1969) and Burton and Miller (1971) and with the development of a wide range of methods for the discretization of the integral operators in the BIE, progress has been made in resolving these difficulties. Further useful information on these formulations and methods can be found in Burton (1973), Kleinmann and Roach (1974), Burton (1976), Kirkup (1989), and Harris (1990). Importan results from the application of BEMs to acoustic problems have been described in Meyer et al. (1978), Terai (1980), Sayh et al. (1981), Seybert et al. (1985), Amini and Wilton (1986), Kirkup (1989), and Harris (1990).

The usual way of solving the problem (1) is to apply the $\mathrm{BEM}$ for each value of $k$ individually. This requires the construction of the discrete forms of the integral operators using numerical integration and the solution of a linear system of equations. Clearly, for a surface divided into a large number of elements the use of the BEM in this way is a major com-
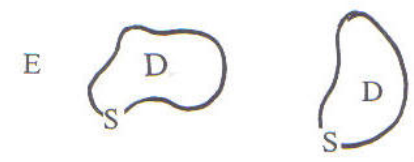

E

Fig. 1 The acoustic radiation model

Transactions of the ASME 
putational task. In this paper the ARM as it is posed by (1)that is the complete problem of solving a large sequence of $\mathrm{HE} 3 \mathrm{~N}$ problems with different wavenumbers and different boundary conditions corresponding to each wavenumber-is considered in order to obtain a computationally more efficient solution. The techniques that are described are for use in the computation of the discrete form of the BIE and in the solution of the linear systems of equations that arise in BEMs derived via collocation. The methods considered in this paper are not applicable to the solution of the Helmholtz equation at one single wavenumber.

The BIE formulation of the HE3N problem (1) that is considered in this paper is the one introduced by Burton and Miller (1971). The use of this underlying formulation ensures a robust BEM at all wavenumbers. The formulation is stated in Section 3 and the corresponding BEM is stated in Section 4. In Section 5 the various ways of speeding up the evaluation of the discrete forms are considered and in Section 6 a method for speeding up the solution to the linear system of equations is described.

Methods from sections 5 and 6 are implemented in a Fortran subroutine in Section 7. The capabilities of the subroutine for the calculation of sound powers and radiation ratios are demonstrated by applying it to appropriate test problems in Section

\section{Formulae for the Acoustic Properties}

From the solution $\varphi$ to (1) at each wavenumber $k$ the (timeharmonic) sound pressure is given by

$$
P(p)=i \rho \omega \varphi(p) \quad(p \in S \cup E),
$$

where $\rho$ is the density of the acoustic medium, $\omega=k c$ and $c$ is the speed of sound in the medium. The acoustic intensity on the surface is

$$
I(p)=\frac{1}{2} \operatorname{Re}\left\{P^{*}(p) v(p)\right\} \quad(p \in S)
$$

where ${ }^{*}$ denotes the complex conjugate. The sound power is given by

$$
W=\int_{S} I(q) d S_{q} .
$$

The radiation ratio (or radiation efficiency) is given by

$$
\sigma_{\mathrm{RAD}}=\frac{W}{\frac{1}{2} \rho c \int_{S} v^{*}(q) v(q) d S_{q}} .
$$

\section{Boundary Integral Equation Formulation}

The BIE on which we base the BEM is the one introduced in Burton and Miller (1971)

$$
\begin{gathered}
\alpha\left\{M_{k} \varphi\right\}(p)-\alpha c(p) \varphi(p)+\beta\left\{N_{k} \varphi\right\}(p)=\alpha\left\{L_{k} \frac{\partial \varphi}{\partial n}\right\}(p) \\
+\beta\left\{M_{k}^{\prime} \frac{\partial \varphi}{\partial n}\right\}(p)+\beta c(p) \frac{\partial \varphi}{\partial n}(p) \quad(p \in S) \\
\varphi(p)=\left\{M_{k} \varphi\right\}(p)-\left\{L_{k} \frac{\partial \varphi}{\partial n}\right\} \quad(p \in E)
\end{gathered}
$$

The $L_{k}, M_{k}, M_{k}{ }^{t}, N_{k}$ in (6) denote integral operators and are defined as follows:

$$
\begin{aligned}
&\left\{L_{k} \mu\right\}(p) \equiv \int_{S} G_{k}(p, q) \mu(q) d S_{q} \quad(p \in D \cup S \cup E), \\
&\left\{M_{k} \mu\right\}(p) \equiv \int_{S} \frac{\partial G_{k}}{\partial n_{q}}(p, q) \mu(q) d S_{q} \quad(p \in D \cup S \cup E),
\end{aligned}
$$

$$
\begin{aligned}
& \left\{M_{k}^{\prime} \mu\right\}(p) \equiv \frac{\partial}{\partial n_{p}} \int_{S} G_{k}(p, q) \mu(q) d S_{q} \quad(p \in S), \\
& \left\{N_{k} \mu\right\}(p) \equiv \frac{\partial}{\partial n_{p}} \int_{S} \frac{\partial G_{k}}{\partial n_{q}}(p, q) \mu(q) d S_{q} \quad(p \in S)
\end{aligned}
$$

Where in (7) $n_{q}, n_{p}$ are the unit outward normals to the surface $S$ at $q, p, \mu(q)$ is a bounded function defined for $q \in S, G_{k}(p, q)$ is the free space Green's function for the Helmholtz equation,

$$
G_{k}(p, q)=\frac{e^{i k r}}{4 \pi r} \text {. }
$$

The function $c(p)$ is defined as follows

$$
c(p)=\left\{\begin{array}{c}
0 \text { when } p \in D, \\
\text { solid angle } / 4 \pi \text { subtended by } \\
\quad \text { the exterior at } q \text { when } q \in S, \\
1 q \in E .
\end{array}\right.
$$

The $\alpha$ and $\beta$ in (6a) are complex numbers with $\alpha \neq 0, \beta \neq 0$, $\operatorname{Im}(\alpha / \beta) \neq 0$. The conditions on $\alpha$ and $\beta$ ensure that the integral equation (6) has a unique solution for all $k$.

\section{Derivation of the Boundary Element Method Through Collocation}

The boundary element method is derived by replacing the surface $S$ by an approximate surface and the boundary func tions by a linear combination of basis functions. The symbol $\tilde{S}$ is used to represent the approximate surface, the symbols $\tilde{D}$ and $\tilde{E}$ are used to represent the approximate interior and exterior regions. The approximate boundary $\tilde{S}$ can be written as the sum of boundary elements,

$$
\tilde{S}=\sum_{j} \Delta_{j} \tilde{S} .
$$

Having replaced $S$ by $\tilde{S}$, the boundary function $\mu(q)$ is replaced by $\tilde{\mu}(q)$ where $q \in \tilde{S}$, and the function $c(q)$ is replaced by $\tilde{c}(q)$.

Similarly, the integral operators $L_{k}, M_{k}, M_{k}^{\prime}$, and $N_{k}$ defined by Eqs. (7) can be replaced by their analogues $\tilde{L}_{k}, \tilde{M}_{k}, \tilde{M}_{k}^{t}$, and $\tilde{N}_{k}$ with respect to the approximate boundary $\tilde{S}$. For example, the $\tilde{L}_{k}$ operator is defined as follows

$$
\left\{\tilde{L_{k}} \tilde{\mu}\right\}(p) \equiv \int_{\bar{S}} G_{k}(p, q) \tilde{\mu}(q) d S_{q},
$$

The purpose of the collocation method is to provide a mechanism for describing an approximation to the boundary functions in discrete form. Collocation of the boundary function $\tilde{\mu}(q)(q \in \tilde{S})$ allows the construction of the discrete form of $\left\{\tilde{L}_{k} \tilde{\mu}\right\}(p),\left\{\tilde{M}_{k} \tilde{\mu}\right\}(p),\left\{\tilde{M}_{k}^{\prime} \tilde{\mu}\right\}(p)$, and $\left\{\tilde{N}_{k} \tilde{\mu}\right\}(p)$. Let $p_{1}, p_{2}$, $\ldots, p_{n} \in \tilde{S}$ be the $n$ collocation points and let $\tilde{\chi}_{1}, \tilde{\chi}_{2}, \ldots, \tilde{\chi}_{n}$ be basis functions with the usual properties

$$
\begin{gathered}
\tilde{\chi}_{i}\left(p_{j}\right)= \begin{cases}1 & \text { when } i=j \\
0 & \text { when } i \neq j,\end{cases} \\
\sum_{j=1}^{n} \tilde{\chi}_{j}(q)=1(q \in \tilde{S}) .
\end{gathered}
$$

The boundary function $\tilde{\mu}(q)$ is approximated as follows

$$
\tilde{\mu}(q)=\sum_{j=1}^{n} \tilde{\mu}\left(p_{j}\right) \tilde{\chi}_{j}(q) \quad(q \in \tilde{S})
$$

The substitution of the approximation (10) into the definitions of the integral operators allows their expression in discrete form. For example the substitution of (10) into (7a) gives

$$
\left\{\tilde{L}_{k} \tilde{\mu}\right\}(p) \simeq \sum_{j=1}^{n}\left[\tilde{\mu}\left(p_{j}\right)\left\{\tilde{L}_{k} \tilde{\chi}_{j}\right\}(p)\right] .
$$


The following notation is adopted

$$
\begin{gathered}
{\left[\mathrm{L}_{k}\right]_{i j}=\left\{\tilde{L}_{k} \tilde{\chi}_{j}\right\}\left(p_{i}\right),} \\
{\left[\mathrm{M}_{k}\right]_{i j}=\left\{\tilde{M}_{k} \tilde{\chi}_{j}\right\}\left(p_{i}\right),} \\
{\left[\mathrm{M}_{k}\right]_{i j}=\left\{\tilde{M}_{k}{ }^{\prime} \tilde{\chi}_{j}\right\}\left(p_{i}\right),} \\
{\left[\mathrm{N}_{k}\right]_{i j}=\left\{\tilde{N}_{k} \tilde{\chi}_{j}\right\}\left(p_{i}\right),} \\
\underline{\mu}=\left[\tilde{\mu}\left(p_{i}\right), \tilde{\mu}\left(p_{2}\right), \ldots, \tilde{\mu}\left(p_{n}\right)\right]^{T} .
\end{gathered}
$$

$\mathrm{L}_{k}, \mathrm{M}_{k}, \mathrm{M}_{k}{ }^{t}$, and $\mathrm{N}_{k}$ are $n \times n$ matrices and $\mu$ is an $n$-vector The BEM for the solution of the ARP takes the following form.

Primary Stage. An approximation $\hat{\varphi}$ to $\varphi$ is obtained by solving the following linear system of equations

$$
\left[\alpha\left(\mathrm{M}_{k}-\mathrm{C}\right)+\beta \mathrm{N}_{k}\right] \hat{\underline{\varphi}}=\left[\alpha \mathrm{L}_{k}+\beta\left(\mathrm{M}_{k}{ }^{t}+\mathrm{C}\right)\right] \underline{\underline{v}} .
$$

where

$$
\begin{aligned}
& C=\operatorname{diag}\left\{\tilde{c}\left(p_{1}\right), \tilde{c}\left(p_{2}\right), \ldots, \tilde{c}\left(p_{n}\right)\right\}, \\
& \varphi=\left[\varphi\left(p_{1}\right), \varphi\left(p_{2}\right), \ldots, \varphi\left(p_{n}\right)\right]^{T}, \\
& \underline{v}=\left[v\left(p_{1}\right), v\left(p_{2}\right), \ldots, v\left(p_{n}\right)\right]^{T} .
\end{aligned}
$$

Secondary Stage. An approximation $\hat{\varphi}(p)$ to $\varphi(p)$ $(p \in \tilde{E} \cup \tilde{S})$ is obtained from the summation

$$
\begin{aligned}
\hat{\varphi}(p)=\frac{1}{\tilde{c}(p)}\left(\sum_{j=1}^{n}\left[\hat{\varphi}_{j}\left\{\tilde{M}_{k} \tilde{\chi}_{j}\right\}(p)\right]\right. \\
\\
\left.\quad-\sum_{j=1}^{n}\left[v_{j}\left\{\tilde{L}_{k} \tilde{x}_{j}\right\}(p)\right]\right) \quad(p \in \tilde{E} \cup \tilde{S}) .
\end{aligned}
$$

Equations (14) and (15) follow straightforwardly from (6a) and $(6 b)$.

\section{The Evaluation of the Discrete Forms}

In this section methods for speeding up the evaluation of the discrete forms of the integral operators (12) are considered. Three different approaches are outlined, two based on series approximation and the third based on interpolation. Of the three the one based on interpolation seems the most promising although it requires more storage of values than the other methods. The method based on interpolation is the one implemented in the subroutine in Section 7.

Taylor Expansion of the $\exp (i k r)$ Term. The $\exp (i k r)$ $(r=|r|, r=p-q)$ term may be expanded in a Taylor series

$$
\exp (i k r)=1+i k r+\frac{1}{2}(i k)^{2} r^{2}+\cdots
$$

The substitution of a truncated form of the series (16) into the definition of the integral operators allows the calculation of discrete form of the integral operators by the summation of a finite series. However, the $i k r$ term may not be small and hence a large number of terms may be required to ensure a satisfactory approximation. An example of this type of approximation is given by Koopman and Benner (1982).

An alternative approach is derived by expanding the exp $(i k r)$ term as

$$
\begin{aligned}
\exp (i k r)=\exp \left(i k r^{\prime}\right)(1+i k(r & \left.-r^{\prime}\right) \\
& \left.+\frac{1}{2}(i k)^{2}\left(r-r^{\prime}\right)^{2}+\cdots\right),
\end{aligned}
$$

where $r^{\prime}=\left|p-q^{\prime}\right|$ and $q^{\prime}$ is a point near to where the basis function is non-zero. For nonaxisymmetric problems it is ensured that the $\left(r-r^{\prime}\right)$ term is small. However, for large values of $k$ the $k\left(r-r^{\prime}\right)$ term may not be small and hence a fairly large number of terms may be required to ensure a satisfactory approximation. This approach will therefore be applicable only to non-axisymmetric problems and only if the values of $k$ of interest are small relative to the size of the elements.
Interpolation of the Discrete Forms. By evaluating the discrete forms $\left\{\tilde{L}_{k} \tilde{\chi}\right\}(p), \quad\left\{\tilde{M}_{k} \tilde{\chi}\right\}(p), \quad\left\{\tilde{M}_{k}^{\prime} \tilde{\chi}\right\}(p)$, and $\left\{\tilde{N}_{k} \tilde{\chi}\right\}(p)$ or any linear combination of the discrete forms at some prescribed values of wavenumber $k$, the values at other wavenumbers can be approximated using interpolation. The success of such a method depends on the number of interpolation points and their position, the nature of the discrete forms as functions of $k$, the method of interpolation and the range of values over which the interpolant is active. The use of interpolation implies that values of the discrete forms must be stored and hence if this method is used in the primary stage of the boundary element method then several matrices will have to be stored.

The full range of values of wavenumber of interest may be divided into subranges. Interpolation followed by the solution of the HE3N problem is then carried out for the values of wavenumber in each subrange individually. In this way the requirement on computer memory is much less than it would be if interpolation is used over the full range directly.

Straightforward polynomial interpolation could be used Since, in such a case, the interpolation points may be selected by the user, the Chebyshev points should perhaps be chosen, thereby seeking to minimize the maximum error. For example, the discrete form $\left\{L_{k} \tilde{\chi}\right\}(p)$ may be approximated for $k \in\left[k_{1}\right.$, $k_{2}$ ] by using the quadratic interpolant

$$
\begin{aligned}
& \left\{\tilde{L}_{k} \tilde{\chi}\right\}(p) \simeq\left\{\tilde{L}_{\gamma} \tilde{\chi}\right\}(p)+\frac{1}{d}(k-\gamma)\left(\left\{\tilde{L}_{\eta} \tilde{\chi}\right\}(p)-\left\{\tilde{L}_{\gamma} \tilde{\chi}\right\}(p)\right) \\
& \left.+\frac{1}{2 d^{2}}(k-\gamma)(k-\eta)\left(\left\{\tilde{L}_{\gamma} \tilde{\chi}\right\}(\mathrm{p})-2\left\{\tilde{L}_{\eta} \tilde{\chi}\right\}(p)\right\}+\left\{\tilde{L}_{\lambda} \tilde{\chi}\right\}(p)\right)
\end{aligned}
$$

where

$$
\begin{aligned}
\gamma & =k_{1}+\frac{1}{4}(2-\sqrt{3})\left(k_{2}-k_{1}\right), \\
\eta & =\frac{1}{2}\left(k_{1}+k_{2}\right), \\
\lambda & =k_{1}+\frac{1}{4}(2+\sqrt{3})\left(k_{2}-k_{1}\right)
\end{aligned}
$$

are the Chebyshev quadratic interpolation points for the interval $\left[k_{1}, k_{2}\right]$ and

$$
d=\eta-\gamma=\lambda-\eta=\frac{1}{4} \sqrt{3}\left(k_{2}-k_{1}\right) .
$$

A similar interpolation can be used on the discrete forms of the other integral operators, or a linear combination of the discrete forms of the integral operators.

The behavior of the discrete forms $\left\{\tilde{L}_{k} \tilde{\chi}\right\}(p),\left\{\tilde{M}_{k} \tilde{\chi}\right\}(p)$, $\left\{\tilde{M}_{k}{ }^{\prime} \tilde{\chi}\right\}(p)$, and $\left\{\tilde{N}_{k} \tilde{\chi}\right\}(p)$, as functions of $k$, is dominated by the $\exp (i k r)$ term in the integrand, which is common to the definition of the discrete form of all the integral operators. For nonaxisymmetric problems, and when each $\tilde{\chi}$ is non-zero on a localized set of elements the general success of the interpolation method can be improved by writing the exp ( $i k r)$ term in the form of the product of $\exp \left(i k r^{\prime}\right)$ and $\exp \left(i k\left(r-r^{\prime}\right)\right)$ where $r^{\prime}$ is the distance from $p$ to a point $q^{\prime}$ near the elements over which $\tilde{\chi}$ takes non-zero values. If $\tilde{\chi}$ is non-zero on a single element then $q^{\prime}$ may be chosen to be at the middle of that element. The situation is illustrated in Fig. 2. This technique

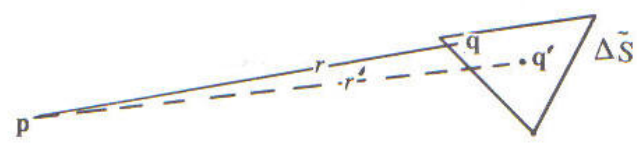

Fig. 2 An illustration of the geometry 
is not applicable to axisymmetric problems since $r-r^{\prime}$ could not then be regarded as small.

For example, the discrete form $\left\{\tilde{L_{k}} \tilde{\chi}\right\}(p)$ may be approximated for $k \in\left[k_{1}, k_{2}\right]$ by using the interpolant

$$
\begin{aligned}
\left\{\tilde{L}_{k} \tilde{\chi}\right\}(p) \simeq e^{i k r^{\prime}}\left[A(p)+\frac{1}{d}(k-\gamma) B(p)\right. & \\
& \left.+\frac{1}{2 d^{2}}(k-\gamma)(k-\eta) C(p)\right]
\end{aligned}
$$

where

$A(p)=e^{-i \gamma r^{\prime}}\left\{\tilde{L_{\gamma}} \tilde{\chi}\right\}(p)$

$B(p)=e^{-i \eta r^{\prime}}\left\{\tilde{L}_{\eta} \tilde{\chi}\right\}(p)-e^{-i \gamma r^{\prime}}\left\{\tilde{L_{\gamma}} \tilde{\chi}\right\}(p)$

$C(p)=e^{-i \gamma r^{\prime}}\left\{\tilde{L}_{\gamma} \tilde{\chi}\right\}(p)-2 e^{-i \eta r^{\prime}}\left\{\tilde{L}_{\eta} \tilde{\chi}\right\}(p)+e^{-i \lambda r^{\prime}}\left\{\tilde{L_{\lambda}} \tilde{\chi}\right\}(p)$

where $\gamma, \eta, \lambda$, and $d$ are as defined by $(18 \mathrm{a}, \mathrm{b})$. Techniques of this latter type have been applied to the Schenck method in Schenck and Benthien (1989).

Estimating the Values of the Discrete Forms from Previously Obtained Values. We seek methods for calculating approximations to $\left\{\tilde{L}_{k} \tilde{\chi}\right\}(p), \quad\left\{\tilde{M}_{k} \tilde{\chi}\right\}(p), \quad\left\{\tilde{M}_{k}^{t} \tilde{\chi}\right\}(p)$, and $\left\{\tilde{N}_{k} \tilde{\chi}\right\}(p)$ for $k=\gamma+\delta$ from their values when $k=\gamma$, where $\delta$ may be regarded as being small. Here we consider only a particular example of this approach.

The exp ( $i k r)$ term is regarded as a function of $k$ and $r$. Let $r^{\prime}$ be the distance between $p$ and a point $q^{\prime}$ near the elements over which $\tilde{\chi}$ is non-zero. Refer back to Fig. 2. We may write $\exp (i(\gamma+\delta) r)=\exp (i \gamma r) \exp \left(i \delta r^{\prime}\right) \exp \left(i \delta\left(r-r^{\prime}\right)\right)$

$$
\simeq \exp (i \gamma r) \exp \left(i \delta r^{\prime}\right)
$$

where in this approximation the $\mathrm{O}\left(\delta\left(r-r^{\prime}\right)\right)$ terms are ig nored, hence the methods considered here are applicable only to nonaxisymmetric problems and when $\tilde{\chi}$ is non-zero on a localized set of elements.

Using this approximation allows us to derive the following approximations:

(i) $\left\{\tilde{L}_{\gamma+\delta} \tilde{\chi}\right\}(p)=\int_{S} \frac{\exp (i(\gamma+\delta) r)}{4 \pi r} \tilde{\chi} d S_{q}$

$$
\begin{aligned}
\simeq \int_{\tilde{S}} \frac{\exp (i \gamma r) \exp \left(i \delta r^{\prime}\right)}{4 \pi r} \tilde{\chi} d S_{q} \\
=\exp \left(i \delta r^{\prime}\right)\left\{\tilde{L}_{\gamma} \tilde{\chi}\right\}(p),
\end{aligned}
$$

(ii) $\left\{\tilde{M}_{\gamma+\delta} \tilde{\chi}\right\}(p)$

$$
\begin{gathered}
=\int_{\bar{S}} \frac{\exp (i(\gamma+\delta) r)}{4 \pi r^{2}}(i(\gamma+\delta) r-1) \frac{\partial r}{\partial n_{q}} \tilde{\chi} d S_{q} \\
\simeq \int_{\bar{S}} \frac{\exp (i \gamma r) \exp \left(i \delta r^{\prime}\right)}{4 \pi r^{2}}(i(\gamma+\delta) r-1) \frac{\partial r}{\partial n_{q}} \tilde{\chi} d S_{q} \\
=\exp \left(i \delta r^{\prime}\right)\left(\left\{\tilde{M}_{\gamma} \tilde{\chi}\right\}(p)+\int_{\tilde{S}}\left(\frac{\exp (i \gamma r)}{4 \pi r^{2}} i \delta r \frac{\partial r}{\partial n_{q}} \tilde{\chi} d S_{q}\right)\right. \\
=\exp \left(i \delta r^{\prime}\right)\left(\left\{\tilde{M}_{\gamma} \tilde{\chi}\right\}(p)+i \delta\left\{\tilde{L}_{\gamma} \tilde{\chi}\right\}(p) \frac{\partial r^{\prime}}{\partial n_{q}^{\prime}}\right),
\end{gathered}
$$

(iii) similarly $\left\{\tilde{M}_{\gamma+\delta}^{t} \tilde{\chi}\right\}(p)$

$$
\approx \exp \left(i \delta r^{\prime}\right)\left(\left\{\tilde{M}_{\gamma}{ }^{\prime} \tilde{\chi}\right\}(p)+i \delta\left\{\tilde{L_{\gamma}} \tilde{\chi}\right\}(p) \frac{\partial r^{\prime}}{\partial n_{p}}\right),
$$

(iv) $\left\{\tilde{N}_{\gamma+\delta} \tilde{\chi}\right\}(p)$

$$
\begin{gathered}
=\int_{\bar{S}} \frac{\exp (i(\gamma+\delta) r)}{4 \pi r^{3}}\left(2-2 i(\gamma+\delta) r-(\gamma+\delta)^{2} r^{2}\right) \frac{\partial r}{\partial n_{q}} \frac{\partial r}{\partial n_{q}} \tilde{\chi} d S_{q} \\
+\int_{\bar{S}} \frac{\exp (i(\gamma+\delta) r)}{4 \pi r^{2}}(i(\gamma+\delta) r-1) \frac{\partial^{2} r}{\partial n_{q} \partial n_{q}} \tilde{\chi} d S_{q}
\end{gathered}
$$

$$
\begin{gathered}
-\exp \left(i \delta r^{\prime}\right)\left(\left\{\tilde{N}_{\gamma} \tilde{\chi}\right\}(p)+\int_{\tilde{S}} \frac{\exp (i \gamma r)}{4 \pi r^{3}}\left(-2 i \delta r-2 \gamma \delta r^{2}\right)\right. \\
\left.\times \frac{\partial r}{\partial n_{p}} \frac{\partial r}{\partial n_{q}} \tilde{\chi} d S_{q}+\int_{\bar{S}} \frac{\exp (i \gamma r)}{4 \pi r^{2}} i \delta r \frac{\partial^{2} r}{\partial n_{p} \partial n_{q}} \tilde{\chi} d S_{q}\right) \\
=\exp \left(i \delta r^{\prime}\right)\left(\left\{\tilde{N}_{\gamma} \tilde{\chi}\right\}(p)+2 i \delta \int_{s} \frac{\exp (i \gamma r)}{4 \pi r} \frac{\partial^{2} r}{\partial n_{p} \partial n_{q}} \tilde{\chi} d S_{q}\right) \\
\simeq \exp \left(i \delta r^{\prime}\right)\left(\left\{\tilde{N}_{\gamma} \tilde{\chi}\right\}(p)+i \delta\left(2\left\{\tilde{M}_{\gamma} \tilde{\chi}\right\}(p) \frac{\partial r^{\prime}}{\partial n_{p}}\right.\right. \\
\left.\left.+\left\{\tilde{L}_{\gamma} \tilde{\chi}\right\}(p) \frac{\partial^{2} r^{\prime}}{\partial n_{p} \partial n_{q}}\right)\right) .
\end{gathered}
$$

The approximation (20) suggest methods for the calculation of the approximations to the discrete forms at one wavenumber from their values at another wavenumber.

\section{The Solution of the Linear System}

In this section an iterative method for the solution of the linear systems of equations that arise is considered. The use of this type of method on the boundary element solution to acoustic radiation problems was originally considered in Amini (1987).

In an $n \times n$ matrix $\mathrm{A}$ has known inverse $\mathrm{A}^{-1}$ then the solution to the linear system

$$
(\mathrm{A}+\mathrm{E}) \underline{x}=\underline{b}
$$

can be found by using the iterative process

$$
\begin{aligned}
\underline{x}_{1} & =\mathrm{A}^{-1} \underline{b} \\
\underline{x}_{j+1} & =\mathrm{A}^{-1} \underline{b}-\mathrm{A}^{-1} \mathrm{E} \underline{x}_{j} \quad j=1,2, \cdots .
\end{aligned}
$$

Alternatively, equivalent information such as the factorization $\mathrm{A}=\mathrm{LU}$ may be known, where $\mathrm{L}$ is lower triangular and $\mathrm{U}$ is upper triangular. The solution to the linear system (21) can then be found by using the iterative process

$$
\begin{array}{ll}
\text { solve for } \underline{x}_{1} & \mathrm{LU} \underline{x}_{1}=\underline{b} \\
\text { solve for } \underline{x}_{j+1} & \mathrm{LU} \underline{x}_{j+1}=\underline{b}-\mathrm{E} \underline{x}_{j} \quad j=1,2, \cdots .
\end{array}
$$

Each iteration in methods $(22,23)$ has an $\mathrm{O}\left(n^{2}\right)$ computational cost. Convergence in the methods $(22,23)$ is assured if $\left\|\mathrm{A}^{-1} \mathrm{E}\right\|<1$ and, in general, the smaller $\left\|\mathrm{A}^{-1} \mathrm{E}\right\|$ the faster the convergence. The method described is the most elementary iterative method for solving a linear system of equations.

The method develops as follows. The assumption that the respective components of the matrices do not vary strongly with $k$ in the range $k \in\left[k_{1}, k_{2}\right]$ is made. The $\alpha$ and $\beta$ parameters are kept constant in the range $\left[k_{1}, k_{2}\right]$. After calculating the inverse (or equivalent) of the matrix that occurs on the left side of the linear system of equations in the primary stage of the BEM for a particular value of $k \in\left[k_{1}, k_{2}\right]$, this can then be used as an approximate inverse for the iterative solution of the linear systems that arise for any $k$ in the range $\left[k_{1}, k_{2}\right]$ using methods $(22,23)$.

\section{Implementation of the Boundary Element Method}

In this section the Fortran subroutine for the solution of the ARP is described. The subroutine is based on the BEM derived from the BIE of Burton and Miller via collocation. The subroutine also employs methods for speeding up the evaluation of the discrete forms and the solution of the linear system. The surfaces $S$, which may take any shape, are described as the sum of a set of $n$ planar triangles $\Delta \tilde{S}_{1}, \Delta \tilde{S}_{2}, \ldots, \Delta \tilde{S}_{n}$. The normal velocity distribution on the surfaces is described by its value at the collocation points $p_{1}, p_{2}, \ldots, p_{n}$, which are the centroids of the triangles.

The basis functions $\tilde{\chi}_{1}, \tilde{\chi}_{2}, \ldots, \tilde{\chi}_{n}$ are the functions 


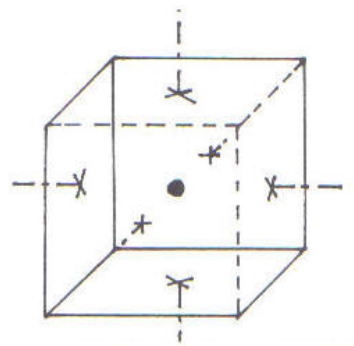

Fig. 3 Illustration of the test problem

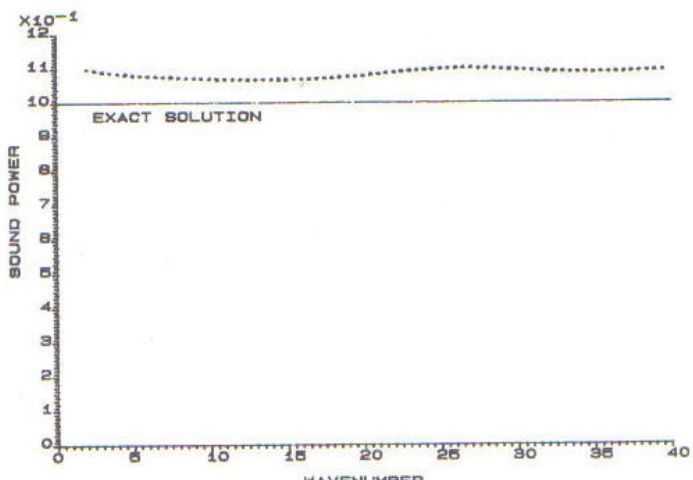

Fig. 4 Comparison of computed and exact sound powers for the cube test problem

$$
\tilde{\chi}_{i}(p)=\left\{\begin{array}{ll}
1 & \text { if } p \in \Delta \tilde{S}_{i} \\
0 & \text { if } p \in \tilde{S}-\Delta \tilde{S}_{i}
\end{array} \text { for } i=1, \ldots, n .\right.
$$

As input, the subroutine accepts a description of the geometry of the surface (made up of triangles), the wavenumbers under consideration, a description of the normal velocity distribution at each wavenumber, and the coordinates of the exterior points of interest. As output, the subroutine gives, for each wavenumber, the acoustic intensity at the centroid of each triangle, the sound power, the radiation ratio, and the sound pressure at the prescribed exterior points.

Methods for speeding up the boundary element solution to the acoustic radiation problem are employed as follows.

(i) The range of wavenumbers is divided into a set of subranges of approximately equal width. The width of each subrange is chosen to be approximately proportional to the reciprocal of the size of the largest element received by the subroutine, although the ratio of the subrange width to the reciprocal of the element size was arrived at only through practical experience with the subroutine.

(ii) For each subrange $\left[k_{1}, k_{2}\right]$ the weighting parameters are calculated by obtaining approximations to the norms of the matrices $M_{k}-\mathrm{I} / 2$ and $\mathrm{N}_{k}$, so that $\alpha \simeq\left\|\mathrm{N}_{k}\right\|_{\infty}$ and $\beta \simeq i \| \mathrm{M}_{k}-\mathrm{I} /$ $2 \|_{\infty}$ for $k=\left(k_{1}+k_{2}\right) / 2$. This is consistent with the method advocated in Kirkup (1991).

(iii) In each subrange $\left[k_{1}, k_{2}\right]$, the matrices with $i j^{\text {th }}$ components

$$
\begin{aligned}
{\left[\mathrm{A}_{k}\right]_{i j}=\exp \left(-i k r\left(p_{i}, p_{j}\right)\right)\left(\alpha\left\{\tilde{M}_{k} \tilde{\chi}_{j}\right\}\right.} & \left(p_{i}\right) \\
& \left.\left.+\beta\left\{\tilde{N}_{k} \tilde{\chi}_{j}\right\}\right)\left(p_{i}\right)\right) \\
{\left[\mathrm{B}_{k}\right]_{i j}=\exp \left(-i k r\left(p_{i}, p_{j}\right)\right)\left(\alpha\left\{\tilde{L}_{k} \tilde{\chi}_{j}\right\}\right.} & \left(p_{i}\right) \\
& \left.\left.+\beta\left\{\tilde{M}_{k}^{t} \tilde{\chi}_{j}\right\}\right)\left(p_{i}\right)\right)
\end{aligned}
$$

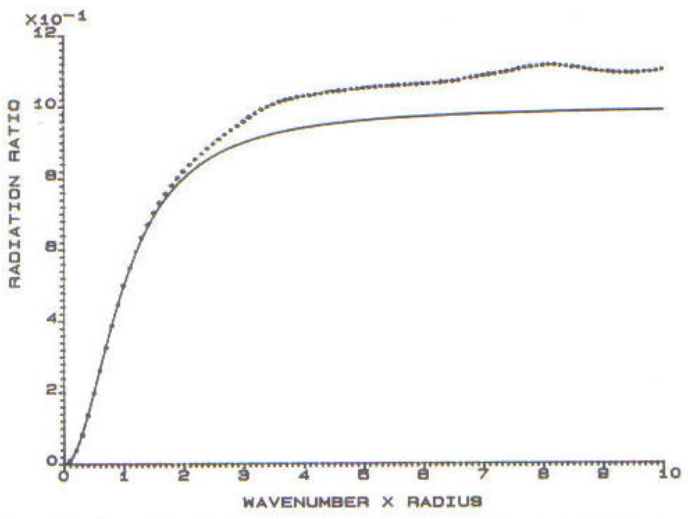

Fig. 5(a) Comparison of the computed and exact sound powers for the pulsating sphere test problem

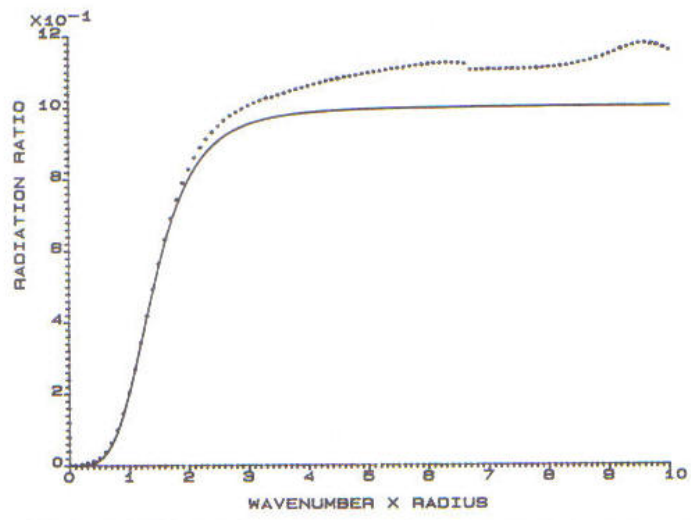

Fig. 5(b) Comparison of the computed and exact sound powers for the oscillating sphere test problem

for $k=\gamma, \eta, \lambda$, where $\gamma, \eta, \lambda$, are as defined by (18a). This gives the matrices $\mathbf{A}_{\gamma}, \mathbf{A}_{\eta}, \mathbf{A}_{\lambda}, \mathbf{B}_{\gamma}, \mathbf{B}_{\eta}, \mathbf{B}_{\lambda}$.

(iv) The inverse of the matrix $\left(\alpha \mathrm{M}_{\eta}-\alpha \mathrm{I} / 2+\beta \mathrm{N}_{\eta}\right)$ is eval uated.

(v) Now, for each $k$ in the range $\left[k_{1}, k_{2}\right]$ approximations to the matrices in (14) can be calculated using

$\left[\alpha \mathrm{M}_{k}-\frac{1}{2} \alpha \mathbf{I}+\beta \mathrm{N}_{k}\right]_{i j} \simeq \mathrm{e}^{i k r^{\prime}}\left(\left[\mathrm{A}_{\gamma}\right]_{i j}\right.$

$$
+\frac{1}{d}(k-\gamma)\left(\left[\mathrm{A}_{\eta}\right]_{i j}-\left[\mathrm{A}_{\gamma}\right\}_{i j}\right)
$$$$
\left.+\frac{1}{2 d^{2}}(k-\gamma)(k-\eta)\left(\left[\mathrm{A}_{\gamma}\right]_{i j}-2\left[\mathrm{~A}_{\eta}\right]_{i j}+\left[\mathrm{A}_{\lambda}\right]_{i j}\right)\right)-\frac{1}{2} \alpha \delta_{i j}
$$

$\left[\alpha \mathrm{L}_{k}+\beta \mathrm{M}_{k}{ }^{l}+\frac{1}{2} \beta \mathrm{I}\right]_{i j} \simeq \mathrm{e}^{i k r^{\prime}}\left(\left[\mathrm{B}_{\gamma}\right]_{i j}+\frac{1}{d}(k-\gamma)\left(\left[\mathrm{B}_{\eta}\right]_{i j}-\left[\mathrm{B}_{\gamma}\right]_{i j}\right)\right.$

$\left.+\frac{1}{2 d^{2}}(k-\gamma)(k-\eta)\left(\left[\mathrm{B}_{\gamma}\right]_{i j}-2\left[\mathrm{~B}_{\eta}\right]_{i j}+\left[\mathrm{B}_{\lambda}\right]_{i j}\right)\right)+\frac{1}{2} \beta \delta_{i j}$

where $d=\eta-\gamma=\lambda-\eta=\sqrt{3}\left(k_{2}-k_{1}\right) / 4$.

(vi) The iterative process (22) is used to solve the linear system (14). 


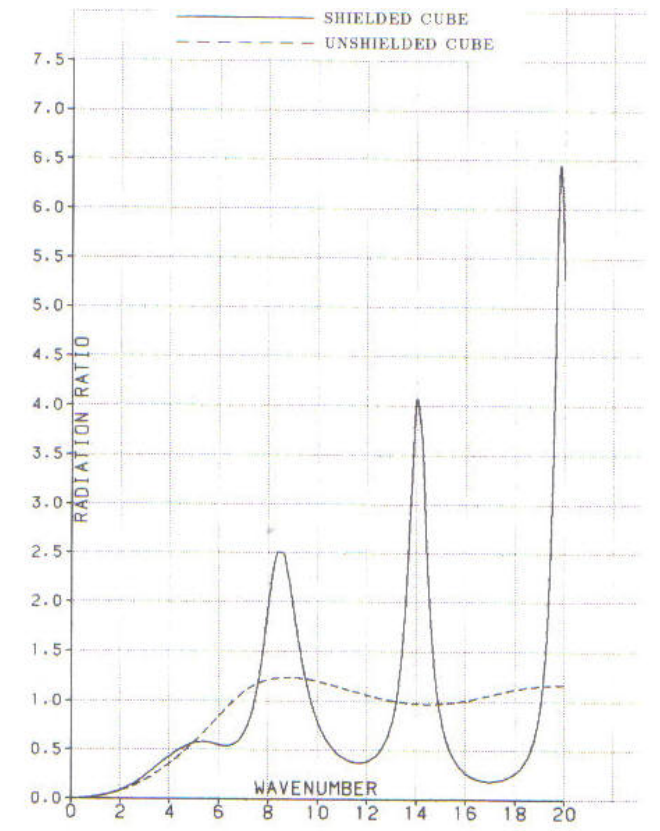

Fig. 6(a) Radiation ratio curves for the cubes distance 0.5 apart and the cube alone

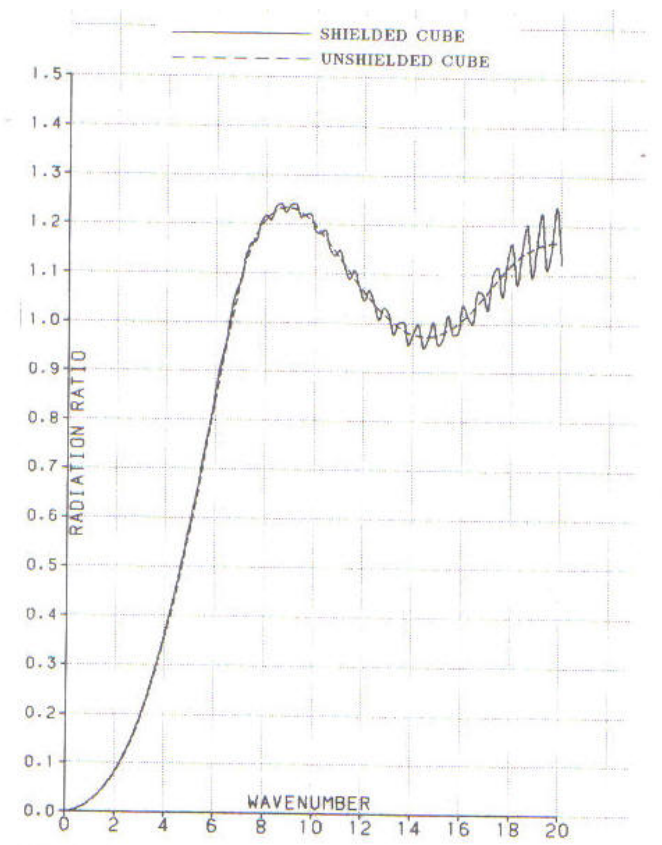
Fig. $6(b)$ Radiation ratio curves for the cubes distance 5.0 apart and
the cube alone
A more complete description of the method employed is given in Kirkup and Henwood (1989).

\section{Results from Test Problems}

In this section we test the method by implementing it on three exterior acoustic problems. Test problems are set up to compute the sound powers and radiation ratios using the subroutine and, where appropriate, comparing the results with exact values.

(1) Sound Powers for a Cube. In the first test problem the surface under consideration is a cube with sides of length 0.2 The test problem is set up by constructing a substitute problem: the surface normal velocity is prescribed to be the values obtained from the point source at the center of the cube, as illustrated in Fig. 3. A point source of unit strength produces a sound power of $k^{2} \rho c / 8 \pi$ where $k$ is the wavenumber. Hence a point source of strength $8 \pi /\left(k^{2} \rho c\right)$ has unit power and it is used as the substitute source in the test. The wavenumbers considered are $k=2.0,2.5,3.0, \ldots, 40.0$. The cube is divided into 96 uniform elements. The comparison between the results obtained from the subroutine and the exact values is given in Fig. 4.

(2) Radiation Ratio Curves for a Pulsating and Oscillating Sphere. In the second test problem the surface under consideration is a sphere of radius $a=0.5$. Two tests are set up in order to compare radiation ratios obtained from the subroutin with the exact radiation ratios. The first test is that of a pulsating sphere, the second test is that of an oscillating sphere. The exact radiation ratio for a pulsating sphere (uniform radia motion) is given by the formula

$$
\sigma_{\mathrm{RAD}}=\frac{(k a)^{2}}{1+(k a)^{2}},
$$

and the radiation ratio for an oscillating sphere (uniform motion in one direction) is given by the formula

$$
\sigma_{\mathrm{RAD}}=\frac{(k a)^{4}}{4+(k a)^{4}}
$$

where $k$ is the wavenumber and $a$ the radius of the sphere, see Morse and Ingard (1968) (pp. 332-336) or Skudrzyk (1971) (pp. 390-391)

The sphere is approximated using 96 triangles of approximately equal size-the approximation to the surface thereby being fairly crude. Figures $5(a)$ and $5(b)$ compare results obtained from the subroutine with exact results for the pulsating and oscillating sphere at the wavenumbers given by $k a=0.1,0.2,0.3, \ldots, 9.9,10.0$.

(3) Radiation Ratio Curves for a Cube with One Vibrating Side Shielded by Another Cube. This test problem consists of two cubes with sides of length 0.5 . The first cube having a side with uniform vibration, the other faces are rigid. The second cube is totally rigid, it stands with one side facing the vibrating side of the first cube. Figures $6(a)$ and $6(b)$ show the radiation ratio curves for the two positions of the cube with vibrating face and shielding face at distances of 0.5 and 5.0 and compare it with the radiation ratio for the situation when there is no second cube. The curves are generated through computing the radiation ratio at wavenumbers $k=0.1,0.2, \ldots$, 20.0 .

Figures 6 show the effect of a shield on the radiation ratio of a vibrating panel on a cube. They show that when the second cube is placed at a distance which is of the same order as the wavelengths under consideration acoustic near-resonances can occur. Figure $6(b)$ shows that if the distance is much greater than the wavelength then the effect of the radiation ratio is small (although weak resonances are evident in the figure). 


\section{Concluding Discussion}

In this paper we have described speed-up methods and have shown how they may be employed in the BEM based on col location of the Burton \& Miller formulation. The method have been tested and results given for systems of both one and two separate surfaces. For acoustic problems with general periodic solutions, the speed-up methods will generally be very beneficial. For example, using quadratic interpolation, as implemented in the subroutine, the discrete forms need be ex plicitly evaluated at three separate frequencies in each band. The use of the iterative method will generally be much less costly than Gaussian elimination-type methods.

Of the methods for speeding up the computation of the discrete form the interpolation method is probably the mos useful. Jumps between neighboring interpolation intervals are apparent only in Fig. $5(b)$. This indicates that the error from the interpolation is small in comparison with the error from other sources such as the effect of approximating the boundary functions and approximating the boundary. From the evidence of the test problems, the iterative method for solving the linear system seemed to work well.

There are many added difficulties with implementing speedup methods. The variation of the matrix elements with wavenumber is dependent on the size of the elements. Hence, since the effectiveness of the interpolation rule and the iterative method are dependent on this, the size of the bands should also be closely related to the size of the elements, as they are in the subroutine. The use of the speed-up method in Section 6 also demands that the choice of parameters $\alpha$ and $\beta$ used remains constant (or is otherwise carefully controlled) throughout each band of wavenumbers. Otherwise one of the assumptions on which the iterative method is based would not hold and the method would not work. Generally it is found that the iterative method works well near the center of the band, whereas convergence is slow near the edges of the band. An analysis of the effectiveness of the iterative method would be useful. It may be possible to construct more successful variants on the iterative method.

\section{Acknowledgment}

The authors are grateful to Dr. G. T. Symm and Mr. G. F. Miller of the National Physical Laboratory, Teddington, Middlesex, United Kingdom for their advice on the work in this paper. The authors are indebted to the reviewers for their helpful comments.

\section{References}

Amini, S., and Wilton, D. T., 1986, "An Investigation of Boundary Element Methods for the Exterior Acoustic Problem," Computer Methods in Applied Mechanics and Engineering, Vol. 54, pp. 49-65.

Amini, S. 1987 " "An Iterative Method for the Boundary Element Solution of the Exterior Acoustic Problem," Journal of Computational and Applied Mathematics, Vol. 20, pp. 109-117,

Burton A. J. and Miller, G. F., 1971 " "The Application of Integral Equation Burton, A.J., and Milier, G. F., 1971, "The Application of Integral Equatio lems," Proc, of the Royal Society London, Vol. A323, pp. 201-210.

Burton, A. J., 1973, "The Solution of Helmholtz' Equation in Exterior DoBurton, A. J., 1973, "The Solution of Helmholtz' Equation in Exterior DoLaboratory, Teddington, Middlesex, U. K.

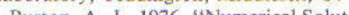

n of Acoustic Radiation Problems," NPL Report OC5/535, National Physical Laboratory, Teddington, Middlesex, J. K.

Harris, P. J., 1990, "Numerical Solution of the Dynamic Fluid-Structure Interaction Problem," PhD thesis, Polytechnic South West U. K.

Kirkup, S. M. 1989, "Solution of Exterior Acoustic Problems by the Bound ary Element Method," PhD thesis, Brighton Polytechnic, U. K.

Kirkup, S. M., and Henwood, D. J., 1989, "Techniques for Speeding-up the Boundary Element Solution of Acoustic Radiation Problems," ASME-WA Symposium on Numerical Techniques in Acoustic Radiation, Bernard, R. J. and Keltie, R. F., eds., pp. 129-137.

Kirkup, S. M., 1991, "On the Choice of Weighting Parameter in the Solution of the Exterior Helmholtz Equation by Improved Boundary Element Methods," Mathematical and Numerical Aspects of Wave Propagation Phenomena, Stras bourg, G. Cohen et al., eds., SIAM, pp. 305-313.

Kleinmann, R. E., and Roach, G. F., 1974, "Boundary Integral Equation for the Three-Dimensional Helmholtz Equation," SIAM Review, Vol. 16, No. 2, pp. 214-235

Koopman, G. H., and Benner, H., 1982, "Method for Computing the Sound Power of Machines based on the Helmholtz Integral," The Journal of the Acoustical Society of America, Vol. 71, No. 1, pp. 78-89.

Kussmaul, R., 1969, "Ein numerisches Verfahren zur Lösung der Neumannschen AuBrenraumproblems für die Helmholtzsche Schwingungsgleichung," Computing, Vol. 4, pp. 246-273.

Meyer, W. L., Bell, W. A., Zinn, B. T., and Stallybrass, M. P., 1978, "Boundary Integral Solutions of Three Dimensional Acoustic Radiation Problems," Journal of Sound and Vibration, Vol. 59, No. 2, pp. 245-262.

Morse, P. M., and Ingard, K. U., 1968, Theoretical Acoustics, McGraw-Hill. Sayhi, M. N., Ousset, Y., and Verchery, G., 1981, "Solution of Radiation Problems by Collocation of Integral Formulations in Terms of Single and Double Layer Potentials," Journal of Sound and Vibration, Vol. 74, No. 2, pp. 187204.

Seybert, A. F., Soenarko, B., Rizzo, F. J., and Shippy, D, J., "An Advanced Computational Method for Radiation and Scattering of Acoustic Waves in Three Dimensions," Journal of the Acoustical Society of America, Vol. 77, pp. 362 368 .

Schenck, H. A., 1968, "Improved Integral Formulations for Acoustic Radiation Problems," Journal of the Acoustical Society of America, Vol. 44, pp. 41-58.

Schenck, H. A., and Benthien, G. W., 1989, "The Application of a Coupled Finite-Element Boundary Element Technique to Large-Scale Structural Acoustic ternational Conference on BEM Cambridge, Massachusetts, Brebbia C. A and Connor, J. J. eds. Vol 2 .

Skudrzyk, E., 1971, The Foundations of Acousties, Springer-Verlag. Terai, T., 1980, "On the Calculation of Sound Fields around Three-Dimenionat Objets by integral Equation Methods," Journal of Sound and Vibration. Vol. 69 , No. 1, pp. $71-100$. 